\title{
Article
}

http://dx.doi.org/10.11646/phytotaxa.172.1.5

\section{The Epimedium wushanense (Berberidaceae) species complex, with one new species from Sichuan, China}

\author{
YANJUN ZHANG ${ }^{1}$, HAISHAN DANG ${ }^{1}$, JIANQIANG LI $^{1, *} \&$ YING WANG ${ }^{1, *}$ \\ 1 Key Laboratory of Plant Germplasm Enhancement and Specialty Agriculture, Wuhan Botanical Garden, Chinese Academy of \\ Sciences, Wuhan, 430074, P. R. China \\ * Authors for correspondence: \\ Jianqiang Li; Tel: 8627 87510330; Fax: 8627 87510251; e-mail: lijq@wbgcas.cn \\ Ying Wang; Tel: 8627 87510675; Fax: 8627 87510331; e-mail: yingwang@wbgcas.cn
}

\begin{abstract}
Epimedium wushanense (Berberidaceae) as treated in the Flora of China includes four species similar in leaflet shape: $E$. wushanense, E. ilicifolium, E. jinchengshanense (sp. nov.), and E. pseudowushanense. Its seven type specimens represent three of the four species. In the present paper, E. wushanense is identified according to morphological characters of its holotype. Except for four of the seven type specimens belonging to $E$. wushanense and E. ilicifolium, the remaining three specimens represent a new species, E. jinchengshanense. Furthermore, E. wushanense from Guangxi and Guizhou as treated in the Flora of China is recognized as E. pseudowushanense. Epimedium wushanense, E. ilicifolium, E. jinchengshanense, and E. pseudowushanense differ by their distributions and flowers. Based on the floral characters, E. jinchengshanense is grouped into ser. Dolichocerae, E. wushanense is adjusted from ser. Dolichocerae to ser. Davidianae, and E. ilicifolium is moved from ser. Davidianae to ser. Dolichocerae.
\end{abstract}

Key words: Berberidaceae, Epimedium wushanense, species complex, revision.

\section{Introduction}

Epimedium L. (1753: 117) is the largest herbaceous genus of the Berberidaceae and contains approximately 58 species distributed disjunctly and very unevenly in the Mediterranean region and eastern Asia (Stearn 2002; Ying et al. 2011). As the diversity center of Epimedium, China possesses about 48 species of the genus which are all endemic except Epimedium koreanum Nakai (1936: 63). In his revision of Epimedium, Stearn (2002) grouped all of China's endemic species into section Diphyllon (Kom.) Stearn (2002: 48), which was divided into four series based mainly on floral morphology, particularly petal characteristics.

Epimedium wushanense T.S. Ying (1975: 55), of sect. Diphyllon, was published based on seven collections from Sichuan (and Chongqing), China. One of the most diagnostic characters of this species is its lanceolate or narrowly lanceolate leaflet. Stearn (2002) proposed E. wushanense with long-spurred petals lacking lamina, classifying it into series Dolichocerae Stearn (1938: 509). In the Flora of China, Ying et al. (2011) listed its distribution as Chongqing, Guangxi, Guizhou, Hubei, and Sichuan; however, E. wushanense from Guangxi and Guizhou was recognized as an insufficiently known species, Epimedium pseudowushanense B.L. Guo (2007: 814). Based on our extensive studies on plants in herbaria, the field, and cultivation, we found that $E$. wushanense described in the Flora of China actually includes four distinct species, which are similar in leaflet shape, with a new species Epimedium jinchengshanense Y.J. Zhang \& J.Q. Li. In the present paper, we revise the E. wushanense species complex and discuss the differences of these four species in their morphology and distribution. 
E. ilicifolium-CHINA. Chongqing: Wuxi, B.L. Guo 8 (IMD), A09 (IMD), P.T. Nee 37 (CDBI). Shaanxi: Langao, Y.J. Zhang 52 (HIB), 54 (HIB), 123 (HIB); Pingli, B.L. Guo A55 (IMD), Y.J. Zhang 113 (HIB), Y.L. Qiao 1133 (KUN); Zhengping, B.L. Guo A53 (IMD), A59 (IMD), A60 (IMD), P.Y. Li 1390 (KUN), 2857 (KUN), Y.J. Zhang 109 (HIB), 110 (HIB), 111 (HIB), 109 (HIB), 110 (HIB), 111 (HIB), 241 (HIB), 243 (HIB).

E. pseudowushanense-CHINA. Guangxi, Longsheng, F.L. Guang 790 (IBK); Rongshui, L. Chen 1973 (HIB), L.K. Pei 0518 (IMD). Guizhou, Congjiang, F.Q. Hu s.n. (HGCM), J.M. Yuan 84 (HGAS); Dushan, G.Z. Fan 4-4 (HGCM), 4-9 (HGCM); Guiding, Q.H. Chen 1687 (HGAS); Leishan, B.L. Guo A68 (IMD), A97 (IMD), Q.H. Chen 3026 (HGAS), 3325 (HGAS), 3598 (HGAS), S. Guizhou Exped. 1389 (PE, HGAS), 1727 (HGAS), S.Z. He 98025 (HGCM), Y.J. Zhang 50 (HIB), 93 (HIB), 95 (HIB), 262 (HIB), 425 (HIB), X.B. Yang 9 (HGCM); Libo, G.Z. Fan 5-13 (HGCM), Q.H. Chen 2377 (HGAS); Liping, Anon. 75-1227 (HGAS), J.M. Yuan 174 (HGAS), 726 (HGAS); Longli, B.L. Guo A39 (IMD); Rongjiang, H.A. Deng 45 (HGCM); Sandu, Q.L. Lin et al. 5 (HGCM); Taijiang, Y.J. Zhang 150 (HIB), 152 (HIB), Z.B. Xu 102 (HGCM).

\section{References}

Guo, B.L., He, S.Z., Zhong, G.Y. \& Xiao, P.G. (2007) Two new species of Epimedium (Berberidaceae) from China. Acta Phytotaxonomica Sinica 45: 813-821.

IUCN (2013) The IUCN red list of threatened species, version 2013.9. IUCN Red List Unit, Cambridge U.K. Available from: http://www. iucnredlist.org/ (accessed: 20 February 2014).

Linnaeus, C. (1753) Species plantarum, 1. Laurentii Salvii, Holmiae, 117 pp.

Nakai, T. (1936) Flora sylvatica Koreana, Part 21. Forest Experimental Station, Keijo, Korea, 63 pp. http://dx.doi.org/10.5962/bhl.title.60644

Stearn W.T. (1938) Epimedium and Vancouveria (Berberidaceae), a monograph. Botanical Journal of the Linnean Society 51: 409-555. http://dx.doi.org/10.1111/j.1095-8339.1937.tb01914.x

Stearn W.T. (1998) Four new Chinese species of Epimedium (Berberidaceae). Kew Bulletin 53: 213-223. http://dx.doi.org/10.2307/4110461

Stearn, W.T. (2002) The genus Epimedium and other herbaceous Berberidaceae. Timber Press, Portland, pp.1-202.

Ying, T.S. (1975) On the Chinese species of Epimedium L. Acta Phytotaxonomica Sinica 13: 49-56.

Ying, T.S., Boufford, D.E. \& Brach, A.R. (2011) Epimedium L. In: Wu, Z.Y., Peter, H. R. \& Hong, D.Y. (eds.) Flora of China. Science Press, Beijing \& Missouri Botanical Garden Press, St. Louis, pp.787-799. 